\title{
Construção e validação de tecnologia educativa para o cuidado domiciliar
}

\author{
Construction and validation of educational technology for home care \\ Construcción y validación de tecnología educativa para el cuidado doméstico
}

Recebido: 03/03/2021 | Revisado: 10/03/2021 | Aceito: 11/03/2021 | Publicado: 20/03/2021

Anderson Araújo Corrêa

ORCID: https://orcid.org/0000-0001-5419-606X Universidade do Estado do Amazonas, Brasil E-mail: andersonaraujo.c1@hotmail.com Lihsieh Marrero

ORCID: https://orcid.org/0000-0002-2856-5682 Universidade do Estado do Amazonas, Brasil E-mail: lmmarrero@uea.edu.br

Gizelia Araújo Cunha Porto

ORCID: https://orcid.org/0000-0001-5363-4646 Universidade Estadual do Maranhão, Brasil

E-mail: gizeliaporto@outlook.com

Dheymi Wilma Ramos Silva

ORCID: https://orcid.org/0000-0002-8716-2939

Universidade Estadual do Maranhão, Brasil

E-mail: dheymiwilma@hotmail.com

Ana Carolina Rodrigues da Silva

ORCID: https://orcid.org/0000-0003-4562-7088

Universidade Federal do Piauí, Brasil

E-mail: enf.carolinarodrigues@hotmail.com

Isiyara Taverna Pimenta

ORCID: https://orcid.org/0000-0003-3090-5444

Fundação Oswaldo Cruz, Brasil

E-mail: isiyarataverna@gmail.com

Jairina Nunes Chaves

ORCID: https://orcid.org/0000-0002-3547-6901

Universidade Estadual do Maranhão, Brasil

E-mail: inanunes@hotmail.com

Otoniel Damasceno Sousa

ORCID: https://orcid.org/0000-0003-3968-2427

Universidade Federal do Piauí, Brasil

E-mail: otho_sousa@hotmail.com

Nathallya Castro Monteiro Alves

ORCID: https://orcid.org/0000-0001-5822-3184

Centro Universitário Luterano de Manaus, Brasil

E-mail: enf.nathallyacastro@hotmail.com

Rayana Gonçalves de Brito

ORCID: https://orcid.org/0000-0002-2933-8887

Fundação Oswaldo Cruz, Brasil

E-mail: rayana-brito@hotmail.com

Francisca Natália Alves Pinheiro

ORCID: https://orcid.org/0000-0002-2443-1871

Universidade Federal do Piauí, Brasil

E-mail: nataliapinheiro@ufpi.edu

\section{Resumo}

Objetivo: construir e validar um e-book sobre cuidados a pacientes em ambiente domiciliar. Metodologia: estudo do tipo metodológico de validação de conteúdo com juízes especialistas, realizada no período de agosto de 2020 a janeiro de 2021, no munícipio de Manaus, Amazonas, Brasil. Aplicou-se um questionário que utiliza a escala do tipo Likert. O instrumento avalia 5 blocos: impressão geral, objetivo, conteúdo, relevância e linguagem. A validação ocorreu via Google Forms. Participaram dez juízes especialistas. A análise quantitativa foi realizada no software Excel para calcular Índice de Validade de Conteúdo. Estipulou-se o valor de $80 \%$ como aceitável para validação. Resultados: o estudo obteve como resultado final a construção e validação do e-book "Manual para o cuidado domiciliar". O produto foi considerado satisfatório, apresentando Índice de Validade de Conteúdo global entre os juízes de 0,94 (94\%). Os juízes sugeriram melhorias em relação a termos técnicos, sendo considerados e modificados para a versão final, que será disponibilizado para inclusão no Programa Melhor em Casa de Manaus. Conclusão: o e-book foi validado quanto ao conteúdo, visual e relevância pelos juízes, obtendo índices satisfatórios acima do valor estipulado. Portanto, constituise em uma tecnologia educativa que pode ser utilizada pelos cuidadores e pacientes inseridos no contexto do programa Melhor em Casa, para promoção de cuidados mais eficazes. 
Palavras-chave: Assistência domiciliar; Educação em saúde; Estudos de validação; Serviços hospitalares de assistência domiciliar; Tecnologia educacional.

\begin{abstract}
Objective: to build and validate an e-book on patient care in the home environment. Methodology: study of the methodological type of content validation with expert judges, carried out from August 2020 to January 2021, in the municipality of Manaus, Amazonas, Brazil. A questionnaire was applied that uses the Likert scale. The instrument assesses 5 blocks: general impression, objective, content, relevance and language. The validation took place via Google Forms. Ten expert judges participated. Quantitative analysis was performed using Excel software to calculate Content Validity Index. The value of $80 \%$ was stipulated as acceptable for validation. Results: the study obtained as a final result the construction and validation of the e-book "Manual for home care". The product was considered satisfactory, with a global Content Validity Index among the judges of 0.94 (94\%). The judges suggested improvements in terms of technical terms, which were considered and modified for the final version, which will be made available for inclusion in the Best at Home in Manaus Program. Conclusion: the e-book was validated in terms of content, visual and relevance by the judges, obtaining satisfactory rates above the stipulated value. Therefore, it is an educational technology that can be used by caregivers and patients in the context of the Best at Home program, to promote more effective care.
\end{abstract}

Keywords: Home nursing; Health education; Validation study; Home care services, Hospital-based; Educational technology.

\title{
Resumen
}

Objetivo: construir y validar un libro electrónico sobre la atención al paciente en el hogar. Metodología: estudio del tipo metodológico de validación de contenido con jueces expertos, realizado de agosto de 2020 a enero de 2021 , en el municipio de Manaus, Amazonas, Brasil. Se aplicó un cuestionario que utiliza la escala Likert. El instrumento evalúa 5 bloques: impresión general, objetivo, contenido, relevancia y lenguaje. La validación se realizó a través de Google Forms. Participaron diez jueces expertos. El análisis cuantitativo se realizó con el software Excel para calcular el índice de validez del contenido. El valor del $80 \%$ se estipuló como aceptable para la validación. Resultados: el estudio obtuvo como resultado final la construcción y validación del libro electrónico "Manual de atención domiciliaria". El producto se consideró satisfactorio, con un índice de validez de contenido global entre los jueces de 0,94 (94\%). Los jueces sugirieron mejoras en términos técnicos, que fueron considerados y modificados para la versión final, que estará disponible para su inclusión en el Programa Best at Home in Manaus. Conclusión: el e-book fue validado en cuanto a contenido, visual y relevancia por los jueces, obteniendo índices satisfactorios por encima del valor estipulado. Por tanto, es una tecnología educativa que pueden utilizar cuidadores y pacientes en el contexto del programa Best at Home, para promover una atención más eficaz.

Palabras clave: Atención domiciliaria de salud; Educación en salud; Estudio de validación; Servicios de atención a domicilio provisto por hospital; Tecnología educacional.

\section{Introdução}

O cuidado domiciliar no Brasil, é uma modalidade de atenção à saúde em plena expansão, abrangendo a assistência em todas as fases da vida, desde a criança até o idoso, oferecendo todos os tipos de cuidado anteriormente restritos aos estabelecimentos de saúde. Nessa perspectiva, é possível afirmar que o cuidado domiciliar rompeu as paredes das unidades prestadoras de serviços de saúde, sendo cada vez mais conhecido e recomendado, oferendo suporte para execução de cuidados básicos, paliativos e intensivos (Consoni, 2015).

A atenção domiciliar remonta do Egito Antigo, mas no Brasil foi introduzido na rede de assistência à saúde oficial pelos médicos de família no século XX. Nas últimas duas décadas esta modalidade de atenção tomou novas proporções, a princípio associadas a visita domiciliar do Programa de Saúde da Família, e mais recentemente com o programa Melhor em Casa. Implementado pela Portaria Ministerial no 2029 de 2011, o programa integra as Redes de Atenção às Urgências e Emergências e oferece suporte a desospitalização de pacientes, entendido como processo de alta hospitalar com cuidado estendido ao domicílio, ofertado por equipes multidisciplinares de atenção domiciliar (EMAD) e equipes multidisciplinares de apoio (EMAP) (Ministério da Saúde, 2012; Neves, Seixas, Andrade \& Castro, 2019).

O cuidado domiciliar está baseado na tríade cuidador/família - paciente - equipe de saúde, sendo uma relação complexa em que todos os componentes interagem na busca do objetivo comum: o cuidar. Todos nesse laço do cuidar têm o seu papel de destaque, seja o paciente exercendo sua autonomia no autocuidado, ou a equipe de saúde na fundamentação do cuidado e execução dentro de normas e protocolos de saúde. Porém, cabe destacar a importância do cuidador/familiar, personagem que, no 
espaço doméstico, realiza e auxilia o paciente nas atividades básicas e essenciais a vida de forma contínua. As atividades do cuidador transpassam o cuidado básico, pois há execução de atividades extradomiciliares de diversas naturezas (Andrade, Silva, Seixas \& Braga, 2017; Mocelin et al., 2017).

Na modalidade de assistência domiciliar a execução de procedimentos técnicos especializados é vital para a saúde do paciente. Diversos procedimentos realizados em ambiente hospitalar, como cateterismo de eliminação vesical, terapia de suporte ventilatório, nutrição enteral e curativos podem ser realizadas no domicílio por um cuidador/familiar não profissional, desde que, devidamente orientados pela equipe de saúde. No domicílio a orientação multiprofissional visa prevenir e diminuir o risco de complicações em relação a execução de procedimentos pelo paciente ou cuidador. Portanto, faz-se necessário a orientação e treinamento em relação aos procedimentos e manutenção de dispositivos na ausência do profissional de saúde (Moura, Rodrigues, Neves \& Oliveira, 2020).

Pelo seu papel central, o cuidador necessita de maior suporte dos profissionais de saúde. Assim, as equipes de saúde devem oferecer suporte baseados no contexto e dinâmica do meio familiar prevendo as condições e limitações de recursos na estrutura domiciliar e/ou financeira, tendo em vista que não permanecem no domicílio em tempo integral e que muitas dessas pessoas acabam assumindo o papel de cuidador de maneira repentina e sem preparo para exercê-lo (Alves et al., 2012; Faria, Aparecido, Cruz \& Khater, 2017).

Insere-se nesse contexto o papel das tecnologias educativas que reúne de forma organizada e sistematizada, os principais conhecimentos da literatura científica especializada, atentando para uma relação didática entre o saber científico e o públicoalvo a quem se destina (Ferreira et al., 2020; Moreira, Sabóia, Camacho, Daher \& Teixeira, 2014).

Este estudo enfoca a necessidade da tecnologia educativa na mediação do ensino entre profissionais de saúde e cuidador/paciente assistidos pelo Programa Melhor em Casa, do Ministério da Saúde do Brasil. A tecnologia deve ser, portanto, entendida como um instrumento facilitador, de auxílio e consulta, por conter informações essenciais ao cuidado em saúde (Rocha, Oliveira, Teixeira \& Nemer, 2019).

O objetivo deste estudo foi construir e validar um e-book sobre cuidados a pacientes em ambiente domiciliar.

\section{Método}

Estudo, de caráter metodológico, com ênfase na construção e validação de conteúdo, por meio de juízes especialistas. A produção da tecnologia educativa foi precedida e fundamentada em uma revisão integrativa da literatura sobre procedimentos de saúde aplicados em ambiente domiciliar. Foram utilizados como base, para a composição da tecnologia educativa, manuais do Ministério da Saúde e livros referências de procedimentos em saúde.

A tecnologia desenvolvida (e-book) é composta por 150 páginas digitadas no software Microsoft PowerPoint versão 2016, em fonte Calibri, tamanho 26, com dimensão de Papel A4 e orientação retrato. As ilustrações foram elaboradas em desenhos e adaptações de imagens à mão livre pelos autores. $\mathrm{O}$ layout foi desenvolvido pelos autores. A versão do material educativo submetida à validação pelos juízes especialistas é intitulada "Manual para o cuidado domiciliar".

O e-book foi estruturado em 11 tópicos de interesse para o público-alvo que versam sobre cuidados domiciliares a pacientes e organização do Programa Melhor em Casa, descritos a seguir: 1. lavagem das mãos, 2. cuidados com traqueostomia, 3. orientações para armazenamento e uso de nutrição enteral, 4. cuidados com gastrostomia, 5. cuidados com sonda nasogástrica e nasoentérica, 6. cuidados com oxigenoterapia, 7. cuidados com a cistostomia, 8. cuidados com sonda vesical de demora, 9. orientações para uso do cateter urinário externo, 10. cuidados com a colostomia, 11. orientações para uso de medicamentos, além da apresentação, organização do Programa Melhor em Casa do município de Manaus, Amazonas e referências.

O estudo foi conduzido entre agosto de 2020 e janeiro de 2021, no âmbito da Escola Superior de Ciências da Saúde da Universidade do Estado do Amazonas, localizada no município de Manaus, Amazonas, Brasil. Foram selecionados juízes 
especialistas baseados nos seguintes critérios de expertise: ter no mínimo titulação de especialista na área da saúde; ter comprovada experiência assistencial na área de saúde domiciliar (mínimo de dois anos); e estar inserido no quadro de funcionários do Programa Melhor em Casa. Para validação consideraram-se as recomendações sobre o número ideal de juízes especialistas, logo participaram do estudo 10 profissionais de saúde (Pasquali, 2009; Coluci, Alexandre \& Milani, 2015).

O convite para participação do esudo foi realizado por meio de contato por aplicativo (WhatsApp). A carta convite descrevia os objetivos e procedimentos para participação do estudo. Àqueles que aceitaram o convite foi encaminhado um link da plataforma online Google Forms que continha o Termo de Consentimento Livre e Esclarecido (TCLE) para leitura e download. Ao assentir o TCLE o juiz especialista teve acesso por link para download da cópia em PDF do e-book e acesso ao instrumento de pesquisa avaliativo (questionário) no Google Forms.

Para a coleta de dados aplicou-se um questionário organizado em formato de escala de Likert com cinco opções de julgamento: "concordo totalmente", "concordo", "discordo", "discordo totalmente" e "indiferente/neutro". As perguntas foram distribuídas em 5 blocos de avaliação: Impressão geral ( 8 perguntas), Objetivo ( 3 perguntas), Conteúdo ( 9 perguntas), Relevância (5 perguntas) e Linguagem (4 perguntas), além de espaços destinados para caracterização do juiz especialista, comentários e sugestões. Atribuiu-se uma nota para cada um dos itens de cada bloco de acordo com as opções de julgamento variando de zero (0) para indiferente/neutro até cinco (5) para concordo totalmente.

Foi utilizado o Índice de Validade de Conteúdo (IVC), que mede a concordância dos juízes quanto à representatividade dos itens em relação ao conteúdo em estudo. O valor de concordância do IVC pode variar de 0,70 a 1,00 (Rocha, Oliveira, Teixeira \& Nemer, 2019). Para o estudo foi adotado o valor 0,80 (80\%) como padrão para estabelecer a excelência da validação. A adoção do valor supracitado vai de encontro com o parâmetro mais utilizado nos estudos divulgados pela Rede de Estudos de Tecnologias Educacionais (Teixeira, 2019).

O IVC foi calculado pelo somatório das respostas concordo totalmente (5) e concordo (4), dividido pelo total de juízes. O IVC global foi obtido através da soma de todos os IVC calculados separadamente, e dividida pelo número de itens.

Os dados obtidos das avaliações dos juízes especialitas foram tabulados por meio do software Microsoft Excel versão 2016 e o tratamento dos dados foram realizados por meio da estatística descritiva.

Os aspectos éticos foram respeitados em todas as etapas do estudo seguindo as disposições da Resolução $n^{\circ} 466 / 12$ do Conselho Nacional de Saúde. O estudo faz parte do macroprojeto "Desenvolvimento e validação de tecnologias interativas em saúde e educação na inovação do cuidado humano" aprovado pelo Comitê de Ética em Pesquisa da Universidade do Estado do Amazonas em 13 de setembro de 2019 sob o parecer n 3.574 .288 e CAAE ${ }^{\circ} 15924919.6 .0000 .5016$.

\section{Resultados}

Participaram da validação 10 juízes especialistas, sendo seis (6) do sexo feminino, com média de idade de 38 anos. Quanto à área de formação, quatro (4) eram enfermeiros, três (3) fisioterapeutas, dois (2) nutricionistas e um (1) fonoaudiólogo. No que se refere a titulação, oito (8) eram especialistas, um (1) mestre, e um (1) doutor. A média do tempo de formação foi de 9,4 anos, mínimo de dois (2) e máximo de 17 anos. Todos trabalhavam na área da assistência domiciliar.

A Tabela 1 apresenta cada item do instrumento de validação, a quantidade de juízes especialistas por item e o IVC por item e o IVC global. Primeiramente, os juízes avaliaram o e-book quanto ao seu layout, tamanho da letra e demais aspectos visuais e estéticos. Apenas um juiz avaliou um item de forma "indiferente/neutro". Verificou-se que, quanto a impressão geral o e-book foi considerado válido, visto que o IVC foi de $95 \%$.

Adiante, os juízes avaliaram o e-book quanto aos blocos de objetivo, conteúdo, relevância e linguagem, todos com aprovação superior a 90\%. Observou-se, com o IVC global calculado, que o $e$-book apresentou avaliação satisfatória em todos os itens investigados, alcançando um valor de $0,94(94 \%)$. 
A análise dos comentários e sugestões demonstrou que 5 juízes sugeriram o acréscimo do tema lesões e curativos como forma de melhorias para o $e$-book. Foram sugeridas ainda a substituição de alguns termos para facilitar a compreensão do públicoalvo.

Tabela 1: Avaliação dos juízes especialistas quanto impressão geral, objetivo, conteúdo, relevância e linguagem do $e$-book, Manaus, Amazonas, Brasil, 2021.

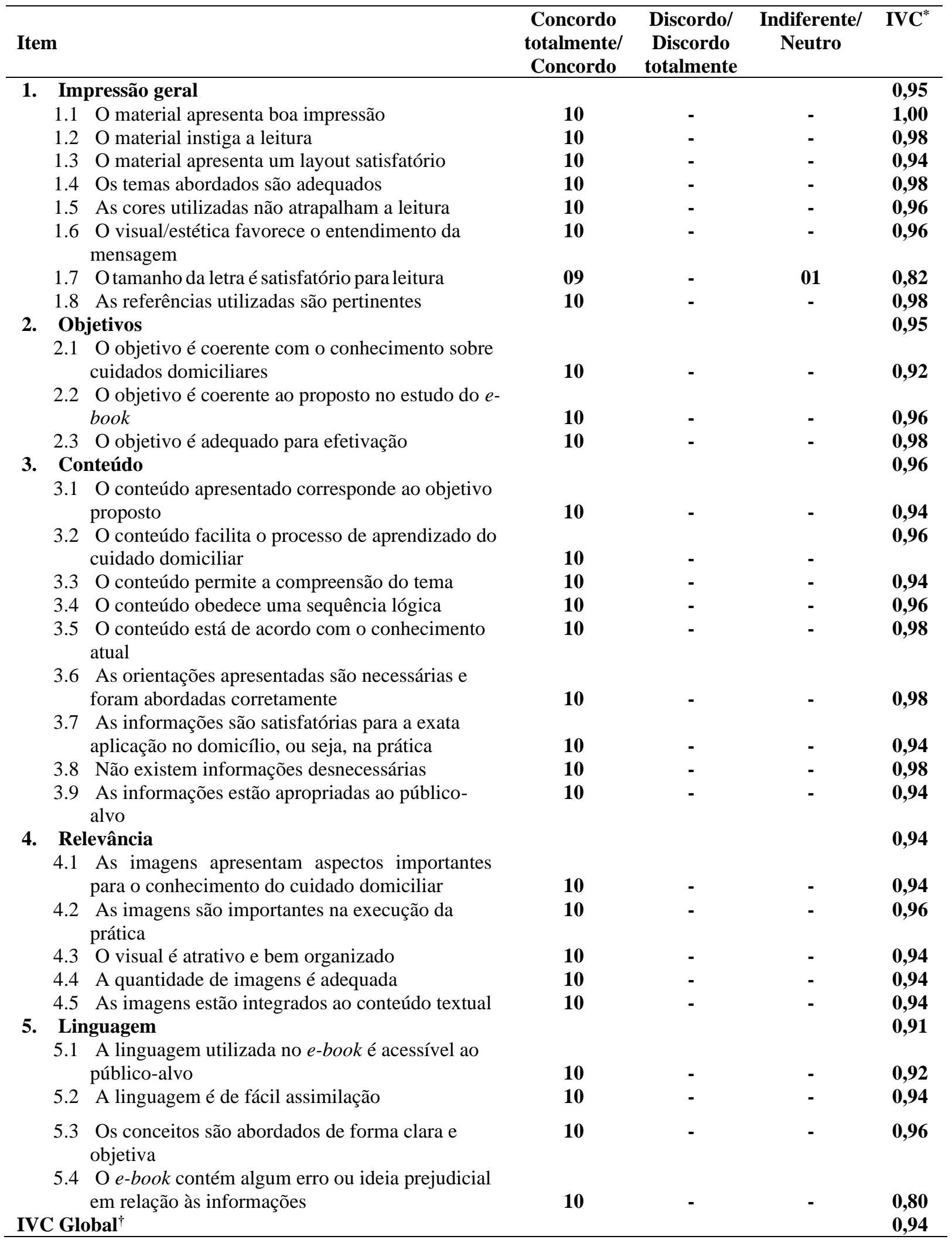




\footnotetext{
* Índice de Validade de Conteúdo.

† soma de todos os Índices de IVC dividida pelo número total de itens do instrumento de validação. Fonte: Autores.
}

Ao final do processo de validação o $e$-book, foi disponibilizado para implementação no Programa Melhor em Casa na cidade de Manaus, Amazonas. Ressalta-se que o mesmo pode ser aplicado em todo o território nacional, atentando para a necessidade de adaptação quanto a estrutura do programa que pode variar entre estados e municípios.

A imagem da capa do $e$-book está representada na Figura 1.

Figura 1: Representação da capa do e-book Manual para o cuidado domiciliar, Manaus, Amazonas, Brasil, 2021.

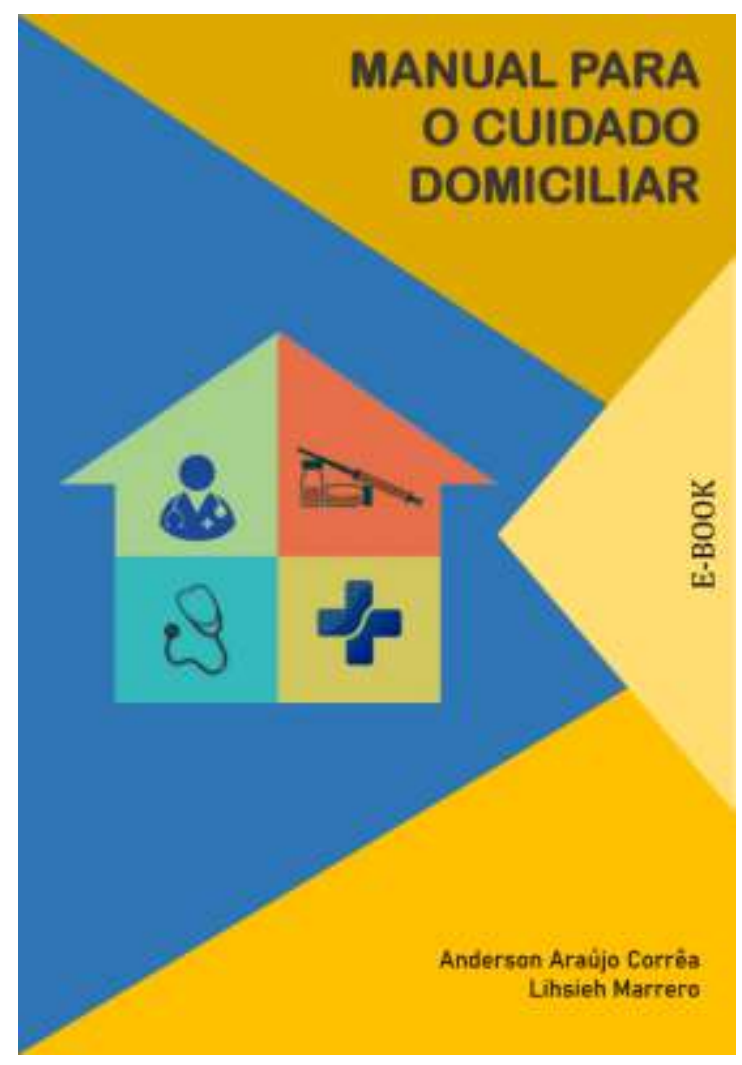

Fonte: Capa do E-book Manual para o cuidado domiciliar.

\section{Discussão}

O processo de validação de materiais educativos é essencial para desenvolver um produto com rigor científico, credibilidade e completude na temática proposta, sendo assim, a validação de conteúdo, realizada por juízes especialistas, é fundamental para o estudo, tendo em vista a adequação e modificação das inconsistências (Medeiros et al., 2015; Lima, Bezerra, Sousa, Rocha \& Oriá 2017; Mello et al., 2020).

No presente estudo, a avaliação do material educativo foi realizada por juízes especialistas de diferentes áreas da saúde. $\mathrm{O}$ intuito foi favorecer a multidisplinaridade, visto que o olhar de diferentes profissionais serve como base para fomentar as orientações da equipe de atendimento domiciliar multiprofissional (Teixeira, 2014; Nascimento \& Teixeira, 2018).

Foi possível analisar, a partir dos resultados do estudo, que o $e$-book "Manual para o cuidado domiciliar" foi avaliado pelos juízes especialistas de forma positiva em relação ao seu visual, objetivo, conteúdo, relevância e linguagem. Foram observados valores de IVC satisfatórios para cada item analisado, assim como, para o IVC global, ambos acima do ponto de corte estabelecido pela literatura (Rocha, Oliveira, Teixeira \& Nemer, 2019). 
O menor IVC (91\%) foi identificado no item "linguagem", sendo os comentários e sugestões dos juízes considerados e inseridos na versão final do e-book, a fim de aprimorar a eficácia do material. Vale destacar que as recomendações dos juízes foram em relação a inclusão de novo tópico, lesões e curativos, além de alterações de alguns termos técnicos para melhor entendimento do público-alvo. Ressalta-se que a inclusão da temática lesões foge da proposta do material construído, visto que a temática requer o acompanhamento contínuo do profissional de enfermagem, além de ser amplo e que requer conhecimentos prévios da literatura e técnicas específicas (Guimarães, Sousa, Coelho \& Galdino, 2017).

Verificou-se que o item "conteúdo" recebeu o maior IVC (96\%) isso significa que o e-book apresenta um embasamento teórico sólido que propicia um processo de aprendizado eficaz, segue ainda uma sequência lógica nas etapas do cuidado para cada procedimento e seu conteúdo condiz com o conhecimento atual. Além disso, todos os juízes concordaram que o e-book apresenta no item "boa impressão" um IVC de 100\%, destaca-se nos comentários a apresentação de figuras adequadas e de fácil compreensão em relação aos materiais necessários ao cuidado domiciliar (Ferreira et al., 2020; Lima, Bezerra, Sousa, Rocha \& Oriá, 2017; Moura et al., 2017).

A utilização de dispositivos móveis como meio de reprodução de tecnologias educativas tem sido promissora. Dentro do contexto multidisciplinar é válida a aplicação de tecnologias que visem promover um cuidado contínuo, visto que a presença do profissional no domicílio não é contínua como no ambiente hospitalar, portanto, a utilização de materiais como apoio do cuidado em saúde vem crescendo ao longo dos últimos anos. Dentro da enfermagem, por exemplo, o uso de tecnologias móveis tem modificado a relação profissional/paciente, pois o uso de tecnologias permite a promoção da saúde e estimula o autocuidado e cuidado por terceiros (Silva et al., 2018).

Destaca-se ainda que a tecnologia e-book é uma alternativa útil e acessível, pois requer apenas o uso de smartphones ou tablet (com leitor de PDF) ou ainda um computador convenvional. Além disso, sua distribuição é eficaz por meio de aplicativos de mensagens ou e-mail. Deve-se levar em consideração a expansão do uso de smartphones como forte aliado na disseminação de materiais educativos com extensão em PDF por apresentar ao usuário facilidade de acesso.

Como limitações do estudo, pode-se citar que, o e-book não contempla todos os procedimentos necessários ao cuidado domiciliar, restringindo-se apenas as principais temáticas vivenciadas pelos profissionais, cuidadores e pacientes. Destaca-se ainda a não inclusão de temas necessários como curativos, procedimentos de fisioterapia e fonoaudiologia, bem como suporte para situação de emergência. Portanto, há diversos temas que devem ser pesquisados e implementados em materiais educativos semelhantes ao desenvolvido. Além disso, é necessária a avaliação deste instrumento por profissionais de saúde que trababalhem fora do contexto do programa no município de Manaus, o que proporcionaria a sua utilização em outros estados e regiões do Brasil. Por fim, a validação pelo público-alvo, cuidadores e pacientes, é necessária para tornar o material mais coeso e aplicável.

\section{Conclusão}

O e-book "Manual para o cuidado domiciliar" foi validado em relação a cinco itens: impressão geral, objetivo, conteúdo, relevância e linguagem por juízes especialistas envolvidos na prática do cuidado domiciliar. Os achados do estudo apontam que o e-book foi considerado válido pelos juízes especialistas ao atingir um IVC global de $94 \%$.

A tecnologia educativa desenvolvida poderá ser inserida na prática de assistência do Programa Melhor em Casa pelos profissionais de saúde durante suas visitas e inclusões de novos pacientes, a fim de reforçar as orientações de procedimentos executados pelo cuidador ou autocuidado pelo paciente, podendo contribuir positivamente na qualidade de vida dos pacientes em atendimento domiciliar.

Espera-se que o $e$-book favoreça a diminuição as intercorrências relacionadas a aplicação de procedimentos de cuidado, pois servirá como manual de orientação, um guia para a prática acurada do cuidado.

Ressalta-se que, a produção desta tecnologia para o cuidado domiciliar não tem o intuito de substituir ou minimizar a 
atuação da equipe profissional, mas apenas servir como suporte para a melhoria do cuidado indicado pelo profissional de saúde, servindo como complementação aos ensinos promovidos pela equipe de saúde.

A disponibilização deste $e$-book, preferencialmente por meio de smartphones, tem potencial para melhorar e fortalecer o processo de trabalho educativo da equipe multiprofissional do Programa Melhor em Casa no município de Manaus.

\section{Agradecimentos}

Agradecemos a todos que direta ou indiretamente contribuíram para a construção deste trabalho, principalmente a Universidade do Estado do Amazonas por meio do Curso de Especialização em Tecnologia e Inovação em Saúde, além do apoio da Samsung Eletrônica da Amazônia LTDA. Somos gratos aos profissionais do Programa Melhor em Casa do município de Manaus que contribuíram, sugeriram e fomentaram o conteúdo desta obra.

\section{Referências}

Alves, S. B., Souza, A. C. S., Tipple, A. F. V., Rezende, K. C. D., Rezende, F. R., \& Rodrigues, É. G. (2012). Manejo de resíduos gerados na assistência domiciliar pela Estratégia de Saúde da Familia. Revista Brasileira de Enfermagem, 65(1), 128-134.

Andrade, A. M., Silva, K. L., Seixas, C. T., \& Braga, P. P. (2017). Nursing practice in home care: an integrative literature review. Revista Brasileira de Enfermagem, 70(1), 210-19.

Coluci, M. Z. O., Alexandre, N. M. C., \& Milani D. (2015). Construction of measurement instruments in the area of health. Ciência \& Saúde Coletiva, 20(3), 925-36.

Consoni E. Os desafios do enfermeiro no cuidado domiciliar. (2015). Enfermagem Brasil, 14(4), 229-34.

Faria, A. A., Aparecido, A. M., Cruz, G. L., \& Khater E. (2017). Cuidando de quem cuida: o papel do psicólogo com cuidadores de pacientes paliativos. (2017). Revista Saúde em Foco, 9, 25-36.

Ferreira, D. S., Teixeira, E., Brown, D. O., Koch, R., Monteiro, W. F., Santos, E. R., \& Oliveira, M. M. C. (2020). Content validation of an educational technology about men's health. Revista Baiana de Enfermagem, 34, e36344.

Guimarães, T. K., Sousa, R. R., Coelho, D. G., \& Galdino, J. H. (2017). Behavior characterization of informal caregivers of wounded patients in the hospital environment. Revista Eletrônica De Enfermagem, 19(28), 1-10.

Lima, A. C. M. A. C. C., Bezerra, K. C., Sousa, D. M. N., Rocha, J. F., \& Oriá, M. O. B. (2017). Development and validation of a booklet for prevention of vertical HIV transmission. Acta Paulista de Enfermagem, 30(2), 181-9.

Medeiros, R. K. S., Ferreira, J. M. A., Pinto, D. P. S. R., Vitor, A. F., Santos, V. E. P., \& Barichello, E. (2015). Modelo de validação de conteúdo de Pasquali nas pesquisas em enfermagem. Revista de Enfermagem Referência, 4(4), 127-35.

Mello, N. C., Góes, F. G. B., Pereira-Ávila, F. M. V., Moraes, J. R. M. M., Silva, L. F., \& Silva, M. A. (2020). Construction and validation of an educational booklet for mobile devices on breastfeeding. Texto \& Contexto - Enfermagem, 29, e20180492.

Ministério da Saúde. (2012). Caderno de atenção domicilia. Ministério da Saúde.

Mocelin, C., Silva, T. G., Celich, K., Madureira, V. F., Souza, S. S., \& Colliselli, L. (2017). The care of dependent elderly on the family contexto. Revista de Pesquisa: Cuidado é Fundamental Online; 9(4), 1034-39.

Moreira, A. P., Sabóia, V. M., Camacho, A. C. L. F., Daher, D. V., \& Teixeira, E. (2014). Educational game of medication administration: a validation study. Revista Brasileira de Enfermagem, 67(4), 528-34.

Moura, I. H., Silva, A. F. R., Rocha, A. E. S. H., Lima, L. H. O., Moreira, T. M. M., \& Silva, A. R. V. (2017). Construction and validation of educational materials for the prevention of metabolic syndrome in adolescents. Revista Latino-Americana de Enfermagem, 25 , e2934.

Moura, T. M., Rodrigues, G. R. S., Neves, V. F. A., \& Oliveira, G. R. S. A. (2020). Invasive procedures on patients under home-care. Revista Enfermagem Contemporânea, $9(1), 85-93$.

Nascimento, M. H. M., \& Teixeira, E. (2018). Educational technology to mediate care of the "kangaroo family" in the neonatal unit. Revista Brasileira de Enfermagem, 71(3), 1290-7.

Neves, A. C. O. J., Seixas, C. T., Andrade, A. M., \& Castro, E. A. B. (2019). Home care: care profile of a service linked to a teaching hospital. Physis: Revista de Saúde Coletiva, 29(2), e290214.

Pasquali, L. (2009). Psychometrics. Revista da Escola de Enfermagem da USP, 43, 992-9.

Rocha, G. S., Oliveira, A. P. P., Teixeira, E., \& Nemer, C. R. B. (2019). Validation of care manual for the elderly after brain surgery. Revista de Enfermagem UFPE on line, 13, e243025. 
Research, Society and Development, v. 10, n. 3, e40410313532, 2021

(CC BY 4.0) | ISSN 2525-3409 | DOI: http://dx.doi.org/10.33448/rsd-v10i3.13532

Silva, A. M. A., Mascarenhas, V. H. A., Araújo, S. N. M., Machado, R. S., Santos, A. M. R., \& Andrade, E. M. L. R. (2018). Mobile technologies in the Nursing area. Revista Brasileira de Enfermagem, 71(5), 2570-8.

Teixeira E. (2019). Desenvolvimento de tecnologias cuidativo-educacionais. Moriá. 